\title{
The second-order asymptotic representation of higher-order non-radial $p$-modes in stars revisited
}

\author{
P. Smeyers
}

Instituut voor Sterrenkunde, Katholieke Universiteit Leuven, Celestijnenlaan 200 B, 3001 Leuven, Belgium

Received 18 November 2005 / Accepted 23 January 2006

ABSTRACT

Aims. We improve the second-order asymptotic theory of higher-order non-radial $p$-modes in spherically symmetric stars that was developed by Smeyers et al. (1996) as an alternative for Tassoul's approach (1990).

Methods. Like the previous authors, we use asymptotic methods appropriate for singular perturbation problems, i.e. expansion procedures in terms of two variables and boundary-layer theory. However, in contrast with them, we no longer adopt boundary-layer coordinates near the singular boundary points that are identical to the fast variable used in the asymptotic expansions at larger distances.

Results. By our definitions of the boundary-layer coordinates, the matchings of the boundary-layer expansions to the asymptotic expansions valid at larger distances from the boundary points, and the constructions of the uniformly valid asymptotic expansions are more transparent. Conclusions. The present asymptotic theory confirms that the application of expansions in terms of two variables and boundary-layer theory to the fourth-order system of differential equations established by Pekeris $(1938$, ApJ, 88, 189) is particularly appropriate for the construction of the asymptotic representation of higher-order $p$-modes in spherically symmetric stars. For these modes, the divergence of the Lagrangian displacement is the basic function, and the radial component of the Lagrangian displacement is of one order higher in the small expansion parameter. In the lowest-order asymptotic approximation, the divergence of the Lagrangian displacement obeys a second-order differential equation of the Sturm-Liouville type. This property explains that the eigenfunction that is associated with the $n$th eigenfrequency displays $n-1$ nodes, with $n=1,2,3, \ldots$

Key words. stars: oscillations - methods: analytical

\section{Introduction}

The second-order asymptotic theory for higher-order nonradial $p$-modes in spherically symmetric stars was first developed by Tassoul (1990). For this purpose, the author went back to a fourth-order system of differential equations composed of two second-order differential equations in the divergence and the radial component of the Lagrangian displacement that was derived many years before by Pekeris (1938). These equations appear to be very convenient for the asymptotic representation of higher-order $p$-modes without the neglect of the Eulerian perturbation of the gravitational potential. For the construction of this representation, Tassoul used basically a method of Olver (1974) that applies to homogeneous second-order differential equations and extended the application of this method to the adopted fourth-order system of differential equations. The procedure requires the preliminary introduction of formal series expansions in terms of Bessel functions for the two dependent variables.

Smeyers et al. (1996) reconsidered the second-order asymptotic theory by using asymptotic methods that apply to singular perturbation problems: expansion procedures in terms of two variables at larger distances from the singular boundary points, and boundary-layer theory near the singular boundary points (Kevorkian \& Cole 1981, 1996). These authors too extended the application of methods described for second-order differential equations to the fourth-order system of differential equations stemming from Pekeris. Smeyers et al. recovered Tassoul's results apart from a minor difference, which did not affect the final results. However, their asymptotic approach matches closer the physical reality that $p$-modes originate from acoustic waves propagating to-and-fro in a resonant cavity inside the star.

The asymptotic treatment of Smeyers et al. has however been obscured by the fact the authors adopted boundary-layer coordinates identical to the fast independent variables that are used in the two-variable expansions at larger distances from the boundary points. Our aim is to improve the asymptotic treatment of Smeyers et al.

The plan of the paper is as follows. In Sect. 2, we recall the basic equations. In Sect. 3, we construct asymptotic expansions at larger distances from the boundary points. We construct boundary-layer expansions near the boundary point at 
$r=0$ and match them to the asymptotic expansions valid at larger distances in Sects. 4 and 5. Similarly, we construct boundary-layer expansions near the boundary point at $r=R$ and match them to the asymptotic expansions valid at larger distances in Sects. 6 and 7.

As a result of the matchings, we derive the eigenfrequency equation in Sect. 8. In Sect. 9, we impose the boundary condition relative to the Eulerian perturbation of the gravitational potential at $r=R$. In Sect. 10, asymptotic expansions that are uniformly valid from the boundary points at $r=0$ and $r=R$ are constructed in their final forms. In Sect. 11, attention is paid to the identification of the radial order of a mode, radial or nonradial, that is associated with a given eigenfrequency. The final section is devoted to concluding remarks.

\section{Basic equations}

Consider a non-rotating spherically symmetric star in hydrostatic equilibrium with mass $M$ and radius $R$ that is subject to a linear, isentropic oscillation depending on time by a factor $\exp (\mathrm{i} \sigma t)$. The normal modes $p, g$, and $f$ belong to a single spherical harmonic $Y_{\ell}^{m}(\theta, \phi)$. With respect to a system of spherical coordinates $r, \theta, \phi$ whose origin coincides with the star's mass centre, the Lagrangian displacement can be represented as

$$
\begin{aligned}
\boldsymbol{\xi}(r, \theta, \phi)= & {\left[\xi_{\ell}(r) \mathbf{1}_{r}\right.} \\
& \left.+\frac{\eta_{\ell}(r)}{r}\left(\mathbf{1}_{\theta} \frac{\partial}{\partial \theta}+\mathbf{1}_{\phi} \frac{1}{\sin \theta} \frac{\partial}{\partial \phi}\right)\right] Y_{\ell}^{m}(\theta, \phi) .
\end{aligned}
$$

Correspondingly, the divergence of the Lagrangian displacement can be expressed as

$$
\begin{aligned}
\alpha(r, \theta, \phi) & =\alpha_{\ell}(r) Y_{\ell}^{m}(\theta, \phi) \\
& \equiv\left[\frac{1}{r^{2}} \frac{\mathrm{d}}{\mathrm{d} r}\left(r^{2} \xi_{\ell}(r)\right)-\frac{\ell(\ell+1)}{r^{2}} \eta_{\ell}(r)\right] Y_{\ell}^{m}(\theta, \phi) .
\end{aligned}
$$

We construct a second-order asymptotic theory for higherorder $p$-modes of a low degree $\ell$ by starting from the fourthorder system of two differential equations for the divergence $\alpha(r)$ and the radial component $\xi(r)$ of the Lagrangian displacement that was established by Pekeris (1938). The system can be written in the form

$$
\begin{aligned}
& \frac{\mathrm{d}^{2} \alpha}{\mathrm{d} r^{2}}+K_{2}(r) \frac{\mathrm{d} \alpha}{\mathrm{d} r}+\left[\frac{\sigma^{2}}{c^{2}}+K_{3}(r)+\frac{K_{1}(r)}{\sigma^{2}}\right] \alpha= \\
& \quad-K_{4}(r) \frac{\mathrm{d} \xi}{\mathrm{d} r}, \\
& \frac{\mathrm{d}^{2} \xi}{\mathrm{d} r^{2}}+\frac{4}{r} \frac{\mathrm{d} \xi}{\mathrm{d} r}-\frac{\ell(\ell+1)-2}{r^{2}} \xi=\frac{\mathrm{d} \alpha}{\mathrm{d} r}+\left[\frac{2}{r}-\frac{1}{\sigma^{2}} \frac{c^{2}}{g} K_{1}(r)\right] \alpha .
\end{aligned}
$$

The variables have their usual meaning, and the coefficients $K_{1}(r), K_{2}(r), K_{3}(r), K_{4}(r)$ are defined as

$$
\begin{aligned}
& K_{1}(r)=\ell(\ell+1) \frac{N^{2}}{r^{2}}, \\
& K_{2}(r)=\frac{2}{r}+\frac{2}{\rho c^{2}} \frac{\mathrm{d}\left(\rho c^{2}\right)}{\mathrm{d} r}-\frac{1}{\rho} \frac{\mathrm{d} \rho}{\mathrm{d} r},
\end{aligned}
$$

$$
\begin{aligned}
K_{3}(r) & =-\frac{\ell(\ell+1)}{r^{2}}+\frac{2 g}{c^{2}}\left(\frac{1}{g} \frac{\mathrm{d} g}{\mathrm{~d} r}+\frac{1}{r}\right) \\
& +\frac{1}{\rho c^{2}} \frac{\mathrm{d}\left(\rho c^{2}\right)}{\mathrm{d} r}\left(\frac{2}{r}-\frac{1}{\rho} \frac{\mathrm{d} \rho}{\mathrm{d} r}\right)+\frac{1}{\rho c^{2}} \frac{\mathrm{d}^{2}\left(\rho c^{2}\right)}{\mathrm{d} r^{2}}, \\
K_{4}(r) & =-\frac{2 g}{c^{2}}\left(\frac{1}{g} \frac{\mathrm{d} g}{\mathrm{~d} r}-\frac{1}{r}\right) .
\end{aligned}
$$

The divergence $\alpha(r)$ and the radial component $\xi(r)$ of the Lagrangian displacement must remain finite both at $r=0$ and at $r=R$. Moreover, at $r=R$, the Eulerian perturbation of the gravitational potential, $\Phi^{\prime}(r)$, and its first derivative, $\mathrm{d} \Phi^{\prime}(r) / \mathrm{d} r$, must satisfy the condition

$$
\left(\frac{\mathrm{d} \Phi^{\prime}}{\mathrm{d} r}\right)_{R}+\frac{\ell+1}{R} \Phi_{R}^{\prime}=-(4 \pi G \rho \xi)_{R} .
$$

We make the differential equations and the boundary condition dimensionless by expressing the time $t$, the radial coordinate $r$, the pressure $P(r)$, the mass density $\rho(r)$, the gravity $g(r)$, the isentropic sound velocity $c(r)$, the gravitational potential $\Phi(r)$, and both the radial component $\xi(r)$ and the transverse component $\eta(r)$ of the Lagrangian displacement respectively in the units $\left[R^{3} /(G M)\right]^{1 / 2}, R, G M^{2} /\left(4 \pi R^{4}\right)$, $M /\left(4 \pi R^{3}\right), G M / R^{2},(G M / R)^{1 / 2}, G M / R, R$. We suppose that the angular frequency $\sigma$ expressed in the unit $\left(G M / R^{3}\right)^{1 / 2}$ is a large quantity and denote it as $1 / \varepsilon$. With this definition, $\varepsilon$ is a small dimensionless quantity that corresponds to the ratio of the oscillation period to $2 \pi$ times the star's dynamic time scale.

In our procedure, we divide the interval $[0, R]$ into three subintervals: the subinterval at larger distances from the singular boundary points at $r=0$ and $r=R$, a boundary layer near the singular boundary point at $r=0$, and a boundary layer near the singular boundary point at $r=R$. The basic principle of the construction of the asymptotic expansions is the same in the three subintervals. From Eq. (3), a homogeneous secondorder differential equation is derived for the lowest-order approximation of the divergence $\alpha(r)$ of the Lagrangian displacement, and from Eq. (4), an inhomogeneous second-order differential equation for the radial component $\xi(r)$ of the Lagrangian displacement. Next, inhomogeneous second-order differential equations are derived successively for the higherorder approximations of $\alpha(r)$ and $\xi(r)$.

\section{Asymptotic expansions at larger distances from the boundary points}

At larger distances from the singular boundary points, Eq. (3) is comparable with a second-order differential equation for a linear oscillator of constant frequency and small damping that is considered by Kevorkian \& Cole (1981, Sect. 3.3.3; 1996, Sect. 4.3.3). Therefore, the asymptotic expansions are constructed by means of the two-variable method.

We introduce the fast independent variable $\tau(r)$ on the ground of the assumption that the asymptotic solutions for $\alpha(r)$ oscillate so rapidly that their second derivatives $\mathrm{d}^{2} \alpha / \mathrm{d} r^{2}$ are of 
the same order of magnitude as the term $\alpha /\left(\varepsilon^{2} c^{2}\right)$, which contains the large parameter. By setting

$\left(\frac{\mathrm{d} \tau}{\mathrm{d} r}\right)^{2}=\frac{1}{\varepsilon^{2} c^{2}(r)}$

and imposing that $\tau(0)=0$, one obtains the positive variable

$\tau(r)=\frac{1}{\varepsilon} \int_{0}^{r} \frac{\mathrm{d} r^{\prime}}{c\left(r^{\prime}\right)}$.

The integral was first introduced by Ledoux (1962) in his asymptotic representation of higher-order radial oscillation modes and corresponds to the time an acoustic wave needs to propagate from the star's centre to the radial distance $r$. As slow independent variable, we use the radial coordinate $r$.

The differential operators in Eqs. (3) and (4) are transformed according to the chain rule, and asymptotic expansions in terms of the two independent variables are introduced for the functions $\alpha(r)$ and $\xi(r)$ as

$$
\left.\begin{array}{c}
\alpha^{(o)}(r ; \varepsilon)=\alpha_{0}^{(o)}(\tau, r)+\varepsilon \alpha_{1}^{(o)}(\tau, r) \\
+\varepsilon^{2} \alpha_{2}^{(o)}(\tau, r) \varepsilon^{3} \alpha_{3}^{(o)}(\tau, r)+O\left(\varepsilon^{4}\right), \\
\xi^{(o)}(r ; \varepsilon)=v(\varepsilon)\left[\xi_{0}^{(o)}(\tau, r)+\varepsilon \xi_{1}^{(o)}(\tau, r)\right. \\
\left.+\varepsilon^{2} \xi_{2}^{(o)}(\tau, r) \varepsilon^{3} \xi_{3}^{(o)}(\tau, r)+O\left(\varepsilon^{4}\right)\right] .
\end{array}\right\}
$$

The function $v(\varepsilon)$ allows to take into account that the order in $\varepsilon$ of the oscillatory part in $\xi^{(o)}(r ; \varepsilon)$ may differ from that of $\alpha^{(o)}(r ; \varepsilon)$.

Equation (3) then takes the form

$$
\begin{aligned}
& \frac{1}{\varepsilon^{2}} \frac{1}{c^{2}}\left(\frac{\partial^{2} \alpha_{0}^{(o)}}{\partial \tau^{2}}+\alpha_{0}^{(o)}\right)+ O\left(\varepsilon^{-1}\right)= \\
& v(\varepsilon)\left[-\frac{1}{\varepsilon} \frac{K_{4}}{c} \frac{\partial \xi_{0}^{(o)}}{\partial \tau}+O\left(\varepsilon^{0}\right)\right] .
\end{aligned}
$$

When $v(\varepsilon)$ is at least of order $\varepsilon^{0}$, the first lowest-order equation is given by the homogeneous differential equation

$\frac{\partial^{2} \alpha_{0}^{(o)}}{\partial \tau^{2}}+\alpha_{0}^{(o)}=0$.

Its general solution

$\alpha_{0}^{(o)}(\tau, r)=A_{0}^{(o)}(r) \cos \tau+B_{0}^{(o)}(r) \sin (\tau)$

involves the yet undetermined functions $A_{0}^{(o)}(r)$ and $B_{0}^{(o)}(r)$.

Next, Eq. (4) takes the form

$\nu(\varepsilon)\left[\frac{1}{\varepsilon^{2}} \frac{1}{c^{2}} \frac{\partial^{2} \xi_{0}^{(o)}}{\partial \tau^{2}}+O\left(\varepsilon^{-1}\right)\right]=\frac{1}{\varepsilon} \frac{1}{c} \frac{\partial \alpha_{0}^{(o)}}{\partial \tau}+O\left(\varepsilon^{0}\right)$.

When $v(\varepsilon)=\varepsilon$, the second lowest-order equation is given by the inhomogeneous differential equation

$\frac{\partial^{2} \xi_{0}^{(o)}}{\partial \tau^{2}}=c \frac{\partial \alpha_{0}^{(o)}}{\partial \tau}$.

Integration yields

$$
\begin{aligned}
\xi_{0}^{(o)}(\tau, r)= & -c(r)\left[B_{0}^{(o)}(r) \cos \tau-A_{0}^{(o)}(r) \sin \tau\right] \\
& +C_{0}^{(o)}(r) \tau+D_{0}^{(o)}(r),
\end{aligned}
$$

where $C_{0}^{(o)}(r)$ en $D_{0}^{(o)}(r)$ are yet undetermined functions. The term $C_{0}^{(o)}(r) \tau$ must be dropped: its order in $\varepsilon$ is inconsistent, since it can be relabeled as $C_{0}^{\prime}(r)$.

At order $\varepsilon^{-1}$, it follows from Eq. (3) that

$$
\begin{aligned}
& \frac{\partial^{2} \alpha_{1}^{(o)}}{\partial \tau^{2}}+\alpha_{1}^{(o)}= \\
& \quad-c\left[2 \frac{\partial^{2} \alpha_{0}^{(o)}}{\partial \tau \partial r}-\left(\frac{1}{2} \frac{1}{c^{2}} \frac{d c^{2}}{d r}-K_{2}\right) \frac{\partial \alpha_{0}^{(o)}}{\partial \tau}\right] .
\end{aligned}
$$

Substitution of the solution for $\alpha_{0}^{(o)}(\tau, r)$ and removal of the resonant terms from the inhomogeneous part of the equation lead to

$A_{0}^{(o)}(r)=A_{0}^{*} h(r), \quad B_{0}^{(o)}(r)=B_{0}^{*} h(r)$,

where $A_{0}^{*}$ and $B_{0}^{*}$ are general constants, and

$h(r)=\left(\rho r^{2} c^{3}\right)^{-1 / 2}$.

The function $h(r)$ corresponds to Tassoul's function $h(r)$.

Hence, the solutions for $\alpha_{0}^{(o)}(\tau, r)$ and $\xi_{0}^{(o)}(\tau, r)$ can be rewritten as

$$
\begin{gathered}
\alpha_{0}^{(o)}(\tau, r)=h(r)\left(A_{0}^{*} \cos \tau+B_{0}^{*} \sin \tau\right), \\
\xi_{0}^{(o)}(\tau, r)=-h(r) c(r)\left(B_{0}^{*} \cos \tau-A_{0}^{*} \sin \tau\right) \\
+D_{0}^{(o)}(r),
\end{gathered}
$$

and Eq. (19) reduces to a homogeneous differential equation with general solution

$\alpha_{1}^{(o)}(\tau, r)=A_{1}^{(o)}(r) \cos \tau+B_{1}^{(o)}(r) \sin \tau$,

where $A_{1}^{(o)}(r)$ and $B_{1}^{(o)}(r)$ are yet undetermined functions.

At order $\varepsilon^{0}$, it follows from Eq. (4) that

$$
\begin{aligned}
\frac{\partial^{2} \xi_{1}^{(o)}}{\partial \tau^{2}}= & c^{2}\left(\frac{\partial \alpha_{0}^{(o)}}{\partial r}+\frac{2}{r} \alpha_{0}^{(o)}\right) \\
& +c\left[\frac{\partial \alpha_{1}^{(o)}}{\partial \tau}-2 \frac{\partial^{2} \xi_{0}^{(o)}}{\partial \tau \partial r}+\left(\frac{1}{2} \frac{1}{c^{2}} \frac{\mathrm{d} c^{2}}{\mathrm{~d} r}-\frac{4}{r}\right) \frac{\partial \xi_{0}^{(o)}}{\partial \tau}\right]
\end{aligned}
$$

After substitution of the appropriate solutions and integration, one obtains

$$
\begin{aligned}
& \xi_{1}^{(o)}(\tau, r)= \\
& \left\{A_{0}^{*} h(r) c(r) \frac{\mathrm{d}}{\mathrm{d} r} \ln \left[h(r) c(r) r^{2}\right]-B_{1}^{(o)}(r)\right\} \\
& c(r) \cos \tau \\
& +\left\{B_{0}^{*} h(r) c(r) \frac{\mathrm{d}}{\mathrm{d} r} \ln \left[h(r) c(r) r^{2}\right]+A_{1}^{(o)}(r)\right\} \\
& c(r) \sin \tau+D_{1}^{(o)}(r),
\end{aligned}
$$

where $D_{1}^{(o)}(r)$ is a yet undetermined function.

Still at order $\varepsilon^{0}$, it follows from Eq. (3) that

$$
\begin{aligned}
\frac{\partial^{2} \alpha_{2}^{(o)}}{\partial \tau^{2}}+\alpha_{2}^{(o)}= & -c\left[2 \frac{\partial^{2} \alpha_{1}^{(o)}}{\partial \tau \partial r}\right. \\
& \left.-\left(\frac{1}{2} \frac{1}{c^{2}} \frac{\mathrm{d} c^{2}}{\mathrm{~d} r}-K_{2}\right) \frac{\partial \alpha_{1}^{(o)}}{\partial \tau}+K_{4} \frac{\partial \xi_{0}^{(o)}}{\partial \tau}\right] \\
& -c^{2}\left(\frac{\partial^{2} \alpha_{0}^{(o)}}{\partial r^{2}}+K_{2} \frac{\partial \alpha_{0}^{(o)}}{\partial r}+K_{3} \alpha_{0}^{(o)}\right)
\end{aligned}
$$


After substitution of the appropriate solutions and introduction with of the function

$W(r)=\frac{1}{h} \frac{\mathrm{d}^{2} h}{\mathrm{~d} r^{2}}+K_{2} \frac{1}{h} \frac{\mathrm{d} h}{\mathrm{~d} r}+\left(K_{3}+K_{4}\right)$

the differential equation becomes

$$
\begin{aligned}
\frac{\partial^{2} \alpha_{2}^{(o)}}{\partial \tau^{2}}+\alpha_{2}^{(o)}= & -\left[2 \frac{\mathrm{d} B_{1}^{(o)}}{\mathrm{d} r}+\left(K_{2}-\frac{1}{c} \frac{\mathrm{d} c}{\mathrm{~d} r}\right) B_{1}^{(o)}\right. \\
& \left.+A_{0}^{*} W(r) h(r) c(r)\right] c \cos \tau \\
& +\left[2 \frac{\mathrm{d} A_{1}^{(o)}}{\mathrm{d} r}+\left(K_{2}-\frac{1}{c} \frac{\mathrm{d} c}{\mathrm{~d} r}\right) A_{1}^{(o)}\right. \\
& \left.-B_{0}^{*} W(r) h(r) c(r)\right] c \sin \tau
\end{aligned}
$$

Removal of the resonant terms from the inhomogeneous part of the differential equation leads to

$$
\left.\begin{array}{l}
A_{1}^{(o)}(r)=h(r)\left[B_{0}^{*} F(r)+A_{1}^{*}\right], \\
B_{1}^{(o)}(r)=-h(r)\left[A_{0}^{*} F(r)-B_{1}^{*}\right] .
\end{array}\right\}
$$

Here $A_{1}^{*}$ and $B_{1}^{*}$ are general constants, and $F(r)$ is a function determined as

$F(r)=\frac{1}{2} \int_{r_{0}}^{r} c\left(r^{\prime}\right) W\left(r^{\prime}\right) \mathrm{d} r^{\prime}$,

where $r_{0}$ is the radial coordinate of a point arbitrarily chosen in the interval $(0,1)$.

The function $W(r)$ has the explicit form

$$
\begin{aligned}
W(r)= & -\frac{\ell(\ell+1)}{r^{2}}+\frac{4}{r} \frac{g}{c^{2}}+\frac{1}{2} \frac{1}{\rho} \frac{\mathrm{d}^{2} \rho}{\mathrm{d} r^{2}} \\
& -\frac{3}{4} \frac{1}{\rho^{2}}\left(\frac{\mathrm{d} \rho}{\mathrm{d} r}\right)^{2}+\frac{1}{r \rho} \frac{\mathrm{d} \rho}{\mathrm{d} r}-\frac{1}{4} \frac{1}{c^{2}}\left(\frac{\mathrm{d} c}{\mathrm{~d} r}\right)^{2}+\frac{1}{2} \frac{1}{c} \frac{\mathrm{d}^{2} c}{\mathrm{~d} r^{2}}
\end{aligned}
$$

and corresponds to Tassoul's function $W_{1}(r)$. For $r \rightarrow 0$,

$c(r) W(r)=-c_{\mathrm{c}} \frac{\ell(\ell+1)}{r^{2}}+O\left(r^{0}\right)$,

and, for $r \rightarrow R$,

$$
\begin{aligned}
c(r) W(r)= & -c_{\mathrm{s}} \frac{\left(2 n_{\mathrm{e}}+1\right)\left(2 n_{\mathrm{e}}+3\right)}{16(R-r)^{3 / 2}} \\
& +O\left((R-r)^{-1 / 2}\right) .
\end{aligned}
$$

Hence, the integrand $c(r) W(r)$ in the definition of the function $F(r)$ becomes infinitely large as $r \rightarrow 0$ and as $r \rightarrow R$.

The solutions for the functions $\alpha_{1}^{(o)}(\tau, r)$ and $\xi_{1}^{(o)}(\tau, r)$ are now

$$
\begin{aligned}
\alpha_{1}^{(o)}(\tau, r)= & h(r)\left\{\left[B_{0}^{*} F(r)+A_{1}^{*}\right] \cos \tau\right. \\
& \left.-\left[A_{0}^{*} F(r)-B_{1}^{*}\right] \sin \tau\right\}, \\
\xi_{1}^{(o)}(\tau, r)= & c(r) h(r)\left\{\left[A_{0}^{*} G(r)-B_{1}^{*}\right] \cos \tau\right. \\
& \left.+\left[B_{0}^{*} G(r)+A_{1}^{*}\right] \sin \tau\right\}+D_{1}^{(o)}(r),
\end{aligned}
$$

$$
G(r)=c(r) \frac{\mathrm{d}}{\mathrm{d} r} \ln \left(r^{2} c(r) h(r)\right)+F(r)
$$

The functions $D_{0}^{(o)}(r)$ and $D_{1}^{(o)}(r)$ in the solutons for $\xi_{0}^{(o)}(\tau, r)$ and $\xi_{1}^{(o)}(\tau, r)$ are still undetermined. In order to determine them, one has to turn to higher-order parts in Eqs. (3) and (4).

As Eq. (28) has become a homogeneous differential equation, its general solution can be written as

$\alpha_{2}^{(o)}(\tau, r)=A_{2}^{(o)}(r) \cos \tau+B_{2}^{(o)}(r) \sin \tau$,

where $A_{2}^{(o)}(r)$ en $B_{2}^{(o)}(r)$ are yet undetermined functions of the slow variable.

At order $\varepsilon$, Eq. (4) leads to a differential equation for $\xi_{2}^{(o)}(\tau, r)$ of the form

$$
\begin{aligned}
& \frac{\partial^{2} \xi_{2}^{(o)}}{\partial \tau^{2}}=H_{1}(r) \cos \tau+H_{2}(r) \sin \tau \\
& \quad-c^{2}\left[\frac{\mathrm{d}^{2} D_{0}^{(o)}}{\mathrm{d} r^{2}}+\frac{4}{r} \frac{\mathrm{d} D_{0}^{(o)}}{\mathrm{d} r}-\frac{\ell(\ell+1)-2}{r^{2}} D_{0}^{(o)}\right] .
\end{aligned}
$$

In order to avoid that the solution for $\xi_{2}^{(o)}(\tau, r)$ contains a mixed secular term, one must set that

$\frac{\mathrm{d}^{2} D_{0}^{(o)}}{\mathrm{d} r^{2}}+\frac{4}{r} \frac{\mathrm{d} D_{0}^{(o)}}{\mathrm{d} r}-\frac{\ell(\ell+1)-2}{r^{2}} D_{0}^{(o)}=0$.

Hence, a general solution for the function $D_{0}^{(o)}(r)$ is given by

$D_{0}^{(o)}(r)=C_{0}^{*} r^{\ell-1}+D_{0}^{*} r^{-(\ell+2)}$,

where $C_{0}^{*}$ en $D_{0}^{*}$ are general constants. The solution for $\xi_{2}^{(o)}(\tau, r)$ then takes the form

$\xi_{2}^{(o)}(\tau, r)=-H_{1}(r) \cos \tau-H_{2}(r) \sin \tau+D_{2}^{(o)}(r)$,

where $D_{2}^{(o)}(r)$ is a yet undetermined function.

Still at order $\varepsilon$, Eq. (3) leads to a differential equation for $\alpha_{3}^{(o)}(\tau, r)$ of the form

$\frac{\mathrm{d}^{2} \alpha_{3}^{(o)}}{\mathrm{d} \tau^{2}}+\alpha_{3}^{(o)}=H_{3}(r) \cos \tau+H_{4}(r) \sin \tau-c^{2} K_{4} \frac{\mathrm{d} D_{0}}{\mathrm{~d} r}$.

In order to remove the resonant terms from the inhomogeneous part, one must set

$H_{3}(r)=0, \quad H_{4}(r)=0$.

A general solution for $\alpha_{3}^{(o)}(\tau, r)$ is then given by

$\alpha_{3}(\tau, r)=A_{3}^{(o)}(r) \cos \tau+B_{3}^{(o)}(r) \sin \tau-c^{2} K_{4} \frac{\mathrm{d} D_{0}}{\mathrm{~d} r}$,

where $A_{3}(r)$ and $B_{3}(r)$ are yet undetermined functions.

Finally, at order $\varepsilon^{2}$, Eq. (4) leads to a differential equation for $\xi_{3}^{(o)}(\tau, r)$ of the form

$$
\begin{aligned}
& \frac{\partial^{2} \xi_{3}^{(o)}}{\partial \tau^{2}}=H_{5}(r) \cos \tau+H_{6}(r) \sin \tau \\
& -c^{2}\left[\frac{\mathrm{d}^{2} D_{1}^{(o)}}{\mathrm{d} r^{2}}+\frac{4}{r} \frac{\mathrm{d} D_{1}^{(o)}}{\mathrm{d} r}-\frac{\ell(\ell+1)-2}{r^{2}} D_{1}^{(o)}\right] .
\end{aligned}
$$


Proceeding as for Eq. (37), one obtains a general solution for $D_{1}^{(o)}(r)$ as

$D_{1}^{(o)}(r)=C_{1}^{*} r^{\ell-1}+D_{1}^{*} r^{-(\ell+2)}$,

where $C_{1}^{*}$ and $D_{1}^{*}$ are general constants.

The second-order asymptotic expansions that are valid at larger distances from the singular boundary points at $r=0$ and $r=R$, can now be written down. When the sums of constants $A_{0}^{*}+\varepsilon A_{1}^{*}, B_{0}^{*}+\varepsilon B_{1}^{*}, C_{0}^{*}+\varepsilon C_{1}^{*}, D_{0}^{*}+\varepsilon D_{1}^{*}$ are renamed respectively as $A_{0}^{*}, B_{0}^{*}, C_{0}^{*}, D_{0}^{*}$, these expansions take the form

$$
\left.\begin{array}{rl}
\alpha^{(o)}(r ; \varepsilon)= & h(r)\left[A_{0}^{*} \cos \tau+B_{0}^{*} \sin \tau\right. \\
& \left.+\varepsilon F(r)\left(B_{0}^{*} \cos \tau-A_{0}^{*} \sin \tau\right)\right]+O\left(\varepsilon^{2}\right), \\
\xi^{(o)}(r ; \varepsilon)= & -\varepsilon c(r) h(r)\left[B_{0}^{*} \cos \tau-A_{0}^{*} \sin \tau\right. \\
& \left.-\varepsilon G(r)\left(A_{0}^{*} \cos \tau+B_{0}^{*} \sin \tau\right)\right] \\
& +\xi^{(o) \text { (n.o.) }}(r ; \varepsilon)+O\left(\varepsilon^{3}\right),
\end{array}\right\}
$$

where $\xi^{(o)(\text { n.o. })}(r ; \varepsilon)$ is the non-oscillatory function of the slow variable

$\xi^{(o)(\text { n.o. })}(r ; \varepsilon)=\varepsilon\left[C_{0}^{*} r^{\ell-1}+D_{0}^{*} r^{-(\ell+2)}\right]$.

The asymptotic expansion $\alpha^{(o)}(r ; \varepsilon)$ is purely oscillatory, while the asymptotic expansion $\xi^{(o)}(r ; \varepsilon)$ consists of both an oscillatory part and a non-oscillatory part. In the asymptotic expansions, the four general constants $A_{0}^{*}, B_{0}^{*}, C_{0}^{*}, D_{0}^{*}$ and the arbitrarily chosen radial coordinate $r_{0}$ appear. The four constants are fixed subsequently by the matchings with the asymptotic expansions valid from the singular boundary points, and the boundary conditions. Furthermore, it will be shown that the choice of the radial coordinate $r_{0}$ has no bearing on the final results.

\section{Boundary-layer expansions near $r=0$}

Some coefficients of the terms in Eqs. (3) and (4) display a pole at the boundary point at $r=0$ or/and at the boundary-point at $r=R$. These terms must be incorporated in the dominant asymptotic equations. Boundary-layer theory is then appropriate for the construction of asymptotic expansions near the singular boundary points. In deriving the dominant boundarylayer equations, we suppose that the term that contains the large parameter $1 / \varepsilon^{2}$ is sufficiently large for becoming of the same order of magnitude as the singular terms somewhere in the boundary layer.

In this section, we concentrate on the construction of boundary-layer expansions near the singular boundary point at $r=0$.
It is convenient to pass on from the function $\xi(r)$ to the function $w(r)$ by means of the transformation

$\xi(r)=c(r) w(r)$

so that Eqs. (3) and (4) become

$$
\begin{gathered}
\frac{\mathrm{d}^{2} \alpha}{\mathrm{d} r^{2}}+K_{2}(r) \frac{\mathrm{d} \alpha}{\mathrm{d} r}+\left(\frac{1}{\varepsilon^{2}} \frac{1}{c^{2}}+K_{3}(r)+\varepsilon^{2} K_{1}(r)\right) \alpha= \\
-K_{4}(r) c\left(\frac{\mathrm{d} w}{\mathrm{~d} r}+\frac{1}{c} \frac{\mathrm{d} c}{\mathrm{~d} r} w\right), \\
\frac{\mathrm{d}^{2} w}{\mathrm{~d} r^{2}}+\left(\frac{4}{r}+\frac{2}{c} \frac{\mathrm{d} c}{\mathrm{~d} r}\right) \frac{\mathrm{d} w}{\mathrm{~d} r} \\
+\left(-\frac{\ell(\ell+1)-2}{r^{2}}+\frac{4}{r} \frac{1}{c} \frac{\mathrm{d} c}{\mathrm{~d} r}+\frac{1}{c} \frac{\mathrm{d}^{2} c}{\mathrm{~d} r^{2}}\right) w= \\
\frac{1}{c} \frac{\mathrm{d} \alpha}{\mathrm{d} r}+\frac{1}{c}\left(\frac{2}{r}-\varepsilon^{2} \frac{c^{2}}{g} K_{1}(r)\right) \alpha .
\end{gathered}
$$

Near $r=0$, the Taylor series hold

$$
\begin{aligned}
c(r) & =c_{c}\left[1+O\left(r^{2}\right)\right], \\
g(r) & =g_{c} r\left[1+O\left(r^{2}\right)\right], \\
K_{1}(r) & =\ell(\ell+1) N_{c}^{2}\left[1+O\left(r^{2}\right)\right], \\
K_{2}(r) & =\frac{2}{r}\left[1+O\left(r^{2}\right)\right], \\
K_{3}(r) & =-\frac{\ell(\ell+1)}{r^{2}}\left[1+O\left(r^{2}\right)\right], \\
K_{4}(r) & =O\left(r^{2}\right) .
\end{aligned}
$$

In the left-hand member of Eq. (49), the second derivative $\mathrm{d}^{2} \alpha / \mathrm{d} r^{2}$ is of the same order in $\varepsilon$ as the singular terms and the term with the large parameter $\alpha /\left(\varepsilon^{2} c^{2}\right)$ when one introduces a boundary-layer coordinate $r^{*}(r)$ which is solution of the equation

$\left(\frac{\mathrm{d} r^{*}}{\mathrm{~d} r}\right)^{2}=\frac{1}{\varepsilon^{2}} \frac{1}{c_{c}^{2}}$

When $r^{*}(0)=0$, a positive boundary-layer coordinate is obtained as

$r^{*}(r)=\frac{1}{\varepsilon} \frac{r}{c_{c}}$

For the functions $\alpha(r)$ and $w(r)$, we introduce boundary-layer expansions of the form

$$
\left.\begin{array}{l}
\alpha^{(c)}(r ; \varepsilon)=\mu_{0}^{(c)}(\varepsilon) \alpha_{0}^{(c)}\left(r^{*}\right)+\mu_{1}^{(c)}(\varepsilon) \alpha_{1}^{(c)}\left(r^{*}\right) \\
\quad+\ldots, \\
w^{(c)}(r ; \varepsilon)=v_{0}^{(c)}(\varepsilon) w_{0}^{(c)}\left(r^{*}\right)+v_{1}^{(c)}(\varepsilon) w_{1}^{(c)}\left(r^{*}\right) \\
\quad+\ldots,
\end{array}\right\}
$$

where $\mu_{0}^{(c)}(\varepsilon), \mu_{1}^{(c)}(\varepsilon), \ldots$ and $v_{0}^{(c)}(\varepsilon), v_{1}^{(c)}(\varepsilon), \ldots$ are asymptotic series to be determined. 
After transformation, Eq. (49) takes the form

$$
\begin{gathered}
\mu_{0}^{(c)}(\varepsilon)\left\{\frac{1}{\varepsilon^{2}}\left[\frac{\mathrm{d}^{2} \alpha_{0}^{(c)}}{\mathrm{d} r^{*^{2}}}+\frac{2}{r^{*}} \frac{\mathrm{d} \alpha_{0}^{(c)}}{\mathrm{d} r^{*}}+\left(1-\frac{\ell(\ell+1)}{r^{* 2}}\right) \alpha_{0}^{(c)}\right]\right. \\
\left.+O\left(\varepsilon^{0}\right)\right\}+\mu_{1}^{(c)}(\varepsilon) \\
\left\{\frac{1}{\varepsilon^{2}}\left[\frac{\mathrm{d}^{2} \alpha_{1}^{(c)}}{\mathrm{d} r^{*^{2}}}+\frac{2}{r^{*}} \frac{\mathrm{d} \alpha_{1}^{(c)}}{\mathrm{d} r^{*}}+\left(1-\frac{\ell(\ell+1)}{r^{* 2}}\right) \alpha_{1}^{(c)}\right]\right. \\
\left.+O\left(\varepsilon^{0}\right)\right\}+\ldots=v_{0}^{(c)}(\varepsilon) O(\varepsilon)+\ldots
\end{gathered}
$$

As $v_{0}^{(c)}(\varepsilon)$ is of a higher order in $\varepsilon$ than $\varepsilon^{-3} \mu_{0}^{(c)}(\varepsilon)$, the first dominant boundary-layer equation is homogeneous and is given by

$$
\frac{\mathrm{d}^{2} \alpha_{0}^{(c)}}{\mathrm{d} r^{*^{2}}}+\frac{2}{r^{*}} \frac{\mathrm{d} \alpha_{0}^{(c)}}{\mathrm{d} r^{*}}+\left[1-\frac{\ell(\ell+1)}{r^{* 2}}\right] \alpha_{0}^{(c)}=0 .
$$

The solution satisfying the requirement that the divergence of the Lagrangian displacement behaves as $r^{\ell}$ as $r \rightarrow 0$, is

$\alpha_{0}^{(c)}\left(r^{*}\right)=A_{0, c} r^{*-1 / 2} J_{\ell+1 / 2}\left(r^{*}\right)$,

where $J_{\ell+1 / 2}\left(r^{*}\right)$ is the Bessel function of the first kind of order $\ell+1 / 2$, and $A_{0, c}$ a general constant.

Next, Eq. (50) takes the form

$$
\begin{aligned}
& v_{0}(\varepsilon)\left\{\frac{1}{\varepsilon^{2}}\right. {\left[\frac{\mathrm{d}^{2} w_{0}^{(c)}}{\mathrm{d} r^{* 2}}+\frac{4}{r^{*}} \frac{\mathrm{d} w_{0}^{(c)}}{\mathrm{d} r^{*}}-\frac{\ell(\ell+1)-2}{r^{* 2}} w_{0}^{(c)}\right] } \\
&\left.+O\left(\varepsilon^{0}\right)\right\} \\
&+v_{1}(\varepsilon)\left\{\frac{1}{\varepsilon^{2}}\left[\frac{\mathrm{d}^{2} w_{1}^{(c)}}{\mathrm{d} r^{* 2}}+\frac{4}{r^{*}} \frac{\mathrm{d} w_{1}^{(c)}}{\mathrm{d} r^{*}}-\frac{\ell(\ell+1)-2}{r^{* 2}} w_{1}^{(c)}\right]\right. \\
&\left.+O\left(\varepsilon^{0}\right)\right\}+\ldots= \\
& \mu_{0}(\varepsilon)\left[\frac{1}{\varepsilon}\left(\frac{\mathrm{d} \alpha_{0}^{(c)}}{\mathrm{d} r^{*}}+\frac{2}{r^{*}} \alpha_{0}^{(c)}\right)+O(\varepsilon)\right] \\
&+\mu_{1}(\varepsilon)\left[\frac{1}{\varepsilon}\left(\frac{\mathrm{d} \alpha_{1}^{(c)}}{\mathrm{d} r^{*}}+\frac{2}{r^{*}} \alpha_{1}^{(c)}\right)+O(\varepsilon)\right]+\ldots
\end{aligned}
$$

As $v_{0}^{(c)}(\varepsilon)=\varepsilon \mu_{0}^{(c)}(\varepsilon)$, the second dominant boundary-layer equation is inhomogeneous and is given by

$$
\frac{\mathrm{d}^{2} w_{0}^{(c)}}{\mathrm{d} r^{* 2}}+\frac{4}{r^{*}} \frac{\mathrm{d} w_{0}^{(c)}}{\mathrm{d} r^{*}}-\frac{\ell(\ell+1)-2}{r^{* 2}} w_{0}^{(c)}=\frac{\mathrm{d} \alpha_{0}^{(c)}}{\mathrm{d} r^{*}}+\frac{2}{r^{*}} \alpha_{0}^{(c)} .
$$

The solution satisfying the requirement that the radial component of the Lagrangian displacement behaves as $r^{\ell-1}$ as $r \rightarrow$ 0 , is

$$
\begin{aligned}
w_{0}^{(c)}\left(r^{*}\right)= & C_{0, c} r^{*(\ell-1)} \\
& +\frac{1}{2 \ell+1}\left[r^{*(\ell-1)} \int_{0}^{r^{*}} r^{\prime-(\ell-2)}\left(\frac{\mathrm{d} \alpha_{0}^{(c)}}{\mathrm{d} r^{\prime}}+\frac{2}{r^{\prime}} \alpha_{0}^{(c)}\right) \mathrm{d} r^{\prime}\right. \\
& \left.-r^{*-(\ell+2)} \int_{0}^{r^{*}} r^{(\ell+3)}\left(\frac{\mathrm{d} \alpha_{0}^{(c)}}{\mathrm{d} r^{\prime}}+\frac{2}{r^{\prime}} \alpha_{0}^{(c)}\right) \mathrm{d} r^{\prime}\right]
\end{aligned}
$$

Here $C_{0, c}$ is a general constant, which has to be set equal to zero as $\ell=0$. The particular solution of the inhomogeneous differential equation can be transformed by a partial integration of the terms that involve the first derivative $\mathrm{d} \alpha_{0}^{(c)}\left(r^{\prime}\right) / \mathrm{d} r^{\prime}$ and by use of the recurrence relations between Bessel functions

$$
\left.\begin{array}{rl}
z^{-(v-1)} J_{v}(z) & =-\frac{\mathrm{d}}{\mathrm{d} z}\left(z^{-(v-1)} J_{v-1}(z)\right) \\
z^{v+1} J_{v}(z) & =\frac{\mathrm{d}}{\mathrm{d} z}\left(z^{v+1} J_{v+1}(z)\right)
\end{array}\right\}
$$

(Abramowitz \& Stegun 1965), so that

$$
\begin{aligned}
w_{0}^{(c)}\left(r^{*}\right)= & C_{0, c} r^{*(\ell-1)}-\frac{A_{0, c}}{2 \ell+1} \\
& \times r^{*-1 / 2}\left[\ell J_{\ell-1 / 2}\left(r^{*}\right)-(\ell+1) J_{\ell+3 / 2}\left(r^{*}\right)\right] .
\end{aligned}
$$

For the determination of the next terms in the boundarylayer expansions, we set $\mu_{1}^{(c)}(\varepsilon)=\varepsilon \mu_{0}^{(c)}(\varepsilon)$ and $v_{1}^{(c)}(\varepsilon)=$ $\varepsilon v_{0}^{(c)}(\varepsilon)$.

From Eq. (55), it then follows that

$\frac{\mathrm{d}^{2} \alpha_{1}^{(c)}}{\mathrm{d} r^{*^{2}}}+\frac{2}{r^{*}} \frac{\mathrm{d} \alpha_{1}^{(c)}}{\mathrm{d} r^{*}}+\left[1-\frac{\ell(\ell+1)}{r^{* 2}}\right] \alpha_{1}^{(c)}=0$,

so that

$\alpha_{1}^{(c)}\left(r^{*}\right)=A_{1, c} r^{*-1 / 2} J_{\ell+1 / 2}\left(r^{*}\right)$,

where $A_{1, c}$ is a general constant.

Next, it follows from Eq. (58) that

$$
\begin{aligned}
\frac{\mathrm{d}^{2} w_{1}^{(c)}}{\mathrm{d} r^{* 2}}+\frac{4}{r^{*}} \frac{\mathrm{d} w_{1}^{(c)}}{\mathrm{d} r^{*}}-\frac{\ell(\ell+1)-2}{r^{* 2}} w_{1}^{(c)} & = \\
& \frac{\mathrm{d} \alpha_{1}^{(c)}}{\mathrm{d} r^{*}}+\frac{2}{r^{*}} \alpha_{1}^{(c)},
\end{aligned}
$$

so that

$$
\begin{aligned}
w_{1}^{(c)}\left(r^{*}\right)= & C_{1, c} r^{*(\ell-1)}-\frac{A_{1, c}}{2 \ell+1} \\
& \times r^{*-1 / 2}\left[\ell J_{\ell-1 / 2}\left(r^{*}\right)-(\ell+1) J_{\ell+3 / 2}\left(r^{*}\right)\right],
\end{aligned}
$$

where $C_{1, c}$ is a general constant.

When one renames the sums of constants $A_{0, c}+\varepsilon A_{1, c}$ and $C_{0, c}+\varepsilon C_{1, c}$ respectively as $A_{0, c}$ and $C_{0, c}$, the boundary-layer expansions from the singular boundary point at $r=0$ take the form

$$
\left.\begin{array}{l}
\alpha^{(c)}(r ; \varepsilon)=\mu_{0}^{(c)}(\varepsilon) A_{0, c} r^{*-1 / 2} J_{\ell+1 / 2}\left(r^{*}\right), \\
w^{(c)}(r ; \varepsilon)=\varepsilon \mu_{0}^{(c)}(\varepsilon)\left\{C_{0, c} r^{*(\ell-1)}-\frac{A_{0, c}}{2 \ell+1} r^{*-1 / 2}\right. \\
\left.\left[\ell J_{\ell-1 / 2}\left(r^{*}\right)-(\ell+1) J_{\ell+3 / 2}\left(r^{*}\right)\right]\right\} .
\end{array}\right\}
$$

The boundary-layer expansions contain the yet undetermined function $\mu_{0}^{(c)}(\varepsilon)$ and the two general constants $A_{0, c}$ and $C_{0, c}$, which are connected with two independent particular solutions of the fourth-order system of equations. With these particular solutions, different functions $\mu_{0}^{(c)}(\varepsilon)$ can be associated. The introduction of distinct functions $\mu_{0}^{(c)}(\varepsilon)$ is suitable for the 
matching of the boundary-layer expansions to the asymptotic expansions that are valid at larger distances from the singular boundary points. The boundary-layer expansions are therefore written in the more general form

$$
\left.\begin{array}{rl}
\alpha^{(c)}(r ; \varepsilon) & =\mu_{0}^{(c, 1)}(\varepsilon) A_{0, c} r^{*-1 / 2} J_{\ell+1 / 2}\left(r^{*}\right), \\
w^{(c)}(r ; \varepsilon) & =\varepsilon \mu_{0}^{(c, 2)}(\varepsilon) C_{0, c} r^{*(\ell-1)}-\varepsilon \mu_{0}^{(c, 1)}(\varepsilon) \\
\frac{A_{0, c}}{2 \ell+1} & r^{*-1 / 2}\left[\ell J_{\ell-1 / 2}\left(r^{*}\right)-(\ell+1) J_{\ell+3 / 2}\left(r^{*}\right)\right] .
\end{array}\right\}
$$

The boundary-layer expansions now involve the two yet undetermined functions $\mu_{0}^{(c, 1)}(\varepsilon)$ and $\mu_{0}^{(c, 2)}(\varepsilon)$, and the two additional general constants $A_{0, c}$ and $C_{0, c}$. Boundary-layer expansion $\alpha^{(c)}(r ; \varepsilon)$ is purely oscillatory, while boundary-layer expansion $w^{(c)}(r ; \varepsilon)$ contains a non-oscillatory part besides its oscillatory part.

\section{Matching of the boundary-layer expansions valid from $r=0$}

The boundary-layer expansions $\alpha^{(c)}(r ; \varepsilon)$ and $\xi^{(c)}(r ; \varepsilon)$ are matched to the asymptotic expansions $\alpha^{(o)}(r ; \varepsilon)$ and $\xi^{(o)}(r ; \varepsilon)$ valid at larger distances from the singular boundary points.

Relative to the divergence of the Lagrangian displacement, the matching condition is

$\lim _{r \rightarrow \infty} \alpha^{(c)}(r ; \varepsilon)=\lim _{r \rightarrow 0} \alpha^{(o)}(r ; \varepsilon)$.

This condition requires an inspection of asymptotic expansion $\alpha^{(o)}(r ; \varepsilon)$ for small values of $r$, and of boundary-layer expansion $\alpha^{(c)}(r ; \varepsilon)$ for large values of $r$.

For small values of $r$, the fast variable $\tau(r)$ tends towards the boundary-layer coordinate $r^{*}(r)$.

The function $F(r)$, which appears in asymptotic expansion $\alpha^{(o)}(r ; \varepsilon)$, can be expressed as

$$
\begin{aligned}
F(r)= & \frac{1}{2} \int_{r_{0}}^{r}\left[c\left(r^{\prime}\right) W\left(r^{\prime}\right)+c_{c} \frac{\ell(\ell+1)}{r^{\prime 2}}\right] \mathrm{d} r^{\prime} \\
& +c_{c} \frac{\ell(\ell+1)}{2 r}-c_{c} \frac{\ell(\ell+1)}{2 r_{0}}
\end{aligned}
$$

and decomposed as

$$
F(r)=F^{(c)}(r)-F^{(c)}\left(r_{0}\right)
$$

with

$$
\begin{aligned}
F^{(c)}(r)=c_{c} & \frac{\ell(\ell+1)}{2 r} \\
+ & \frac{1}{2} \int_{0}^{r}\left[c\left(r^{\prime}\right) W\left(r^{\prime}\right)+c_{c} \frac{\ell(\ell+1)}{r^{\prime 2}}\right] \mathrm{d} r^{\prime} .
\end{aligned}
$$

For small values of $r$, it follows that

$$
F(r)=c_{c} \frac{\ell(\ell+1)}{2 r}-F^{(c)}\left(r_{0}\right)+O(r) .
$$

Considering that, for small values of $r$, as well

$h(r)=\frac{h_{c}}{r}[1+O(r)]$, one obtains

$$
\begin{aligned}
& \lim _{r \rightarrow 0} \alpha^{(o)}(r ; \varepsilon)=\frac{h_{c}}{r}\left\{A_{0}^{*} \cos r^{*}+B_{0}^{*} \sin r^{*}\right. \\
& \left.\quad+\varepsilon\left[c_{c} \frac{\ell(\ell+1)}{2 r}-F^{(c)}\left(r_{0}\right)\right]\left(B_{0}^{*} \cos r^{*}-A_{0}^{*} \sin r^{*}\right)\right\} .
\end{aligned}
$$

On the other hand, one has

$$
\begin{aligned}
\lim _{r \rightarrow \infty} \alpha^{(c)}(r ; \varepsilon) & =\mu_{0}^{(c, 1)}(\varepsilon) \varepsilon A_{0, c}\left(\frac{2}{\pi}\right)^{1 / 2} \frac{c_{c}}{r} \\
\times & {\left[\sin \left(r^{*}-\frac{\ell \pi}{2}\right)+\varepsilon c_{c} \frac{\ell(\ell+1)}{2 r} \cos \left(r^{*}-\frac{\ell \pi}{2}\right)\right] . }
\end{aligned}
$$

A matching of the boundary-layer expansion $\alpha^{(c)}(r ; \varepsilon)$ to the asymptotic expansion $\alpha^{(o)}(r ; \varepsilon)$ is possible, when

$\mu_{0}^{(c, 1)}(\varepsilon)=\varepsilon^{-1}$

and the coefficients of $\cos r^{*}$ and $\sin r^{*}$ are identically zero, so that

$$
\left.\begin{array}{c}
A_{0}^{*}+\varepsilon\left[c_{c} \frac{\ell(\ell+1)}{2 r}-F^{(c)}\left(r_{0}\right)\right] B_{0}^{*}=-A_{0, c} \\
\left(\frac{2}{\pi}\right)^{1 / 2} \frac{c_{c}}{h_{c}}\left(\sin \frac{\ell \pi}{2}-\varepsilon c_{c} \frac{\ell(\ell+1)}{2 r} \cos \frac{\ell \pi}{2}\right), \\
B_{0}^{*}-\varepsilon\left[c_{c} \frac{\ell(\ell+1)}{2 r}-F^{(c)}\left(r_{0}\right)\right] A_{0}^{*}=A_{0, c} \\
\left(\frac{2}{\pi}\right)^{1 / 2} \frac{c_{c}}{h_{c}}\left(\cos \frac{\ell \pi}{2}+\varepsilon c_{c} \frac{\ell(\ell+1)}{2 r} \sin \frac{\ell \pi}{2}\right) .
\end{array}\right\}
$$

As, at order $\varepsilon^{0}$,

$$
\left.\begin{array}{l}
A_{0}^{*}=-A_{0, c}\left(\frac{2}{\pi}\right)^{1 / 2} \frac{c_{c}}{h_{c}} \sin \frac{\ell \pi}{2}, \\
B_{0}^{*}=A_{0, c}\left(\frac{2}{\pi}\right)^{1 / 2} \frac{c_{c}}{h_{c}} \cos \frac{\ell \pi}{2},
\end{array}\right\}
$$

Eqs. (78) reduce to

$$
\left.\begin{array}{l}
A_{0}^{*}=-A_{0, c} \\
\left(\frac{2}{\pi}\right)^{1 / 2} \frac{c_{c}}{h_{c}}\left(\sin \frac{\ell \pi}{2}-\varepsilon F^{(c)}\left(r_{0}\right) \cos \frac{\ell \pi}{2}\right), \\
B_{0}^{*}=A_{0, c} \\
\left(\frac{2}{\pi}\right)^{1 / 2} \frac{c_{c}}{h_{c}}\left(\cos \frac{\ell \pi}{2}+\varepsilon F^{(c)}\left(r_{0}\right) \sin \frac{\ell \pi}{2}\right) .
\end{array}\right\}
$$

By the matching of the boundary-layer expansion $\alpha^{(c)}(r ; \varepsilon)$ to the asymptotic expansion $\alpha^{(o)}(r ; \varepsilon)$, the oscillatory parts of the boundary-layer expansion $\xi^{(c)}(r ; \varepsilon)$ and the asymptotic expansion $\xi^{(o)}(r ; \varepsilon)$ are matched at the same time. The nonoscillatory part of the asymptotic expansion $\xi^{(o)}(r ; \varepsilon)$ tends to that of the boundary-layer expansion $\xi^{(c)}(r ; \varepsilon)$ as $r \rightarrow 0$, when

$D_{0}^{*}=0$

and

$\mu_{0}^{(c, 2)}(\varepsilon)=\varepsilon^{\ell-1}, \quad C_{0}^{*}=c_{c}^{-(\ell-2)} C_{0, c}$. 
From the matching, it results that the functions $\mu_{0}^{(c, 1)}(\varepsilon)$ and $\mu_{0}^{(c, 2)}(\varepsilon)$ are determined, the constants $A_{0}^{*}$ and $B_{0}^{*}$ are related to the constant $A_{0, c}$, the constant $C_{0}^{*}$ is related to the constant $C_{0, c}$, and the constant $D_{0}^{*}$ is equal to zero. The constants $A_{0, c}$ and $C_{0, c}$ remain undetermined.

\section{Boundary-layer expansions near $r=R$}

In the boundary layer near $r=R$, it is appropriate to use $z=$ $R-r$ as the independent variable. In the supposition that the mass density is analytic at the boundary point at $r=R$, one can use a Taylor series of the form

$\rho(r)=\rho_{\mathrm{s}} z^{n_{\mathrm{e}}}[1+O(z)]$

where $n_{\mathrm{e}}$ is a constant. In the approximation in which $m(r) \simeq$ $M$, the Taylor series of the form hold

$$
\left.\begin{array}{rl}
c(r) & =c_{s} z^{1 / 2}[1+O(z)], \\
g(r) & =g_{s}[1+O(z)], \\
K_{1}(r) & =\frac{K_{1, s}}{z}[1+O(z)], \\
K_{2}(r) & =-\left(n_{\mathrm{e}}+2\right) \frac{1}{z}[1+O(z)], \\
K_{3}(r) & =\frac{K_{3, s}}{z}[1+O(z)], \\
K_{4}(r) & =\frac{K_{4, s}}{z}[1+O(z)],
\end{array}\right\}
$$

where $c_{s}$ is positive.

The boundary-layer coordinate $z^{*}(z)$ is determined by the differential equation

$$
\left(\frac{\mathrm{d} z^{*}}{\mathrm{~d} z}\right)^{2}=\frac{1}{\varepsilon^{2}} \frac{1}{c_{s}^{2} z}
$$

When $z^{*}(0)=0$, the positive boundary-layer coordinate is given by

$$
z^{*}(z)=\frac{1}{\varepsilon} \frac{2}{c_{s}} z^{1 / 2}
$$

For the functions $\alpha(r)$ and $w(r)$, we introduce boundary-layer expansions of the form

$$
\begin{aligned}
& \alpha^{(s)}(r ; \varepsilon)=\mu_{0}^{(s)}(\varepsilon) \alpha_{0}^{(s)}\left(z^{*}\right)+\mu_{1}^{(s)}(\varepsilon) \alpha_{1}^{(s)}\left(z^{*}\right) \\
& \quad+\ldots, \\
& w^{(s)}(r ; \varepsilon)=v_{0}^{(s)}(\varepsilon) w_{0}^{(s)}\left(z^{*}\right)+v_{1}^{(s)}(\varepsilon) w_{1}^{(s)}\left(z^{*}\right) \\
& \quad+\ldots,
\end{aligned}
$$

where $\mu_{0}^{(s)}(\varepsilon), \mu_{1}^{(s)}(\varepsilon), \ldots$ and $v_{0}^{(s)}(\varepsilon), v_{1}^{(s)}(\varepsilon), \ldots$ are asymptotic series to be determined.
Equation (49) now takes the form

$$
\begin{aligned}
\mu_{0}^{(s)}(\varepsilon)[ & \frac{1}{\varepsilon^{4}}\left(\frac{\mathrm{d}^{2} \alpha_{0}^{(s)}}{\mathrm{d} z^{* 2}}+\frac{2 n_{\mathrm{e}}+3}{z^{*}} \frac{\mathrm{d} \alpha_{0}^{(s)}}{\mathrm{d} z^{*}}+\alpha_{0}^{(s)}\right) \\
& \left.+O\left(\varepsilon^{-2}\right)\right] \\
+\mu_{1}^{(s)}(\varepsilon)[ & {\left[\frac{1}{\varepsilon^{4}}\left(\frac{\mathrm{d}^{2} \alpha_{1}^{(s)}}{\mathrm{d} z^{* 2}}+\frac{2 n_{\mathrm{e}}+3}{z^{*}} \frac{\mathrm{d} \alpha_{1}^{(s)}}{\mathrm{d} z^{*}}+\alpha_{1}^{(s)}\right)\right.} \\
& \left.+O\left(\varepsilon^{-2}\right)\right]+\ldots=v_{0}^{(s)}(\varepsilon) O\left(\varepsilon^{-3}\right)+\ldots
\end{aligned}
$$

As $v_{0}^{(s)}(\varepsilon)$ is at least of the same order in $\varepsilon$ as $\mu_{0}^{(s)}(\varepsilon)$, the first dominant boundary-layer equation is homogeneous and is given by

$\frac{\mathrm{d}^{2} \alpha_{0}^{(s)}}{\mathrm{d} z^{* 2}}+\frac{2 n_{\mathrm{e}}+3}{z^{*}} \frac{\mathrm{d} \alpha_{0}^{(s)}}{\mathrm{d} z^{*}}+\alpha_{0}^{(s)}=0$.

The solution satisfying the requirement that the divergence of the Lagrangian displacement remains finite at $r=R$, is

$\alpha_{0}^{(s)}\left(z^{*}\right)=A_{0, s} z^{*-\left(n_{\mathrm{e}}+1\right)} J_{n_{\mathrm{e}}+1}\left(z^{*}\right)$,

where $A_{0, s}$ is a general constant.

Next, Eq. (50) takes the form

$$
\begin{gathered}
v_{0}^{(s)}(\varepsilon)\left[\frac{1}{\varepsilon^{4}}\left(\frac{\mathrm{d}^{2} w_{0}^{(s)}}{\mathrm{d} z^{* 2}}+\frac{1}{z^{*}} \frac{\mathrm{d} w_{0}^{(s)}}{\mathrm{d} z^{*}}-\frac{1}{z^{* 2}} w_{0}^{(s)}\right)\right. \\
\left.+O\left(\varepsilon^{-2}\right)\right] \\
+v_{1}^{(s)}(\varepsilon)\left[\frac{1}{\varepsilon^{4}}\left(\frac{\mathrm{d}^{2} w_{1}^{(s)}}{\mathrm{d} z^{* 2}}+\frac{1}{z^{*}} \frac{\mathrm{d} w_{1}^{(s)}}{\mathrm{d} z^{*}}-\frac{1}{z^{* 2}} w_{1}^{(s)}\right)\right. \\
\left.+O\left(\varepsilon^{-2}\right)\right]+\ldots= \\
\mu_{0}^{(s)}(\varepsilon)\left[-\frac{1}{\varepsilon^{3}} \frac{\mathrm{d} \alpha_{0}^{(s)}}{\mathrm{d} z^{*}}+O\left(\varepsilon^{-1}\right)\right] \\
+\mu_{1}^{(s)}(\varepsilon)\left[-\frac{1}{\varepsilon^{3}} \frac{\mathrm{d} \alpha_{1}^{(s)}}{\mathrm{d} z^{*}}+O\left(\varepsilon^{-1}\right)\right]+\ldots
\end{gathered}
$$

As $v_{0}^{(s)}(\varepsilon)=\varepsilon \mu_{0}^{(s)}(\varepsilon)$, the second dominant boundary-layer equation is inhomogeneous and is given by

$\frac{\mathrm{d}^{2} w_{0}^{(s)}}{\mathrm{d} z^{* 2}}+\frac{1}{z^{*}} \frac{\mathrm{d} w_{0}^{(s)}}{\mathrm{d} z^{*}}-\frac{1}{z^{* 2}} w_{0}^{(s)}=-\frac{\mathrm{d} \alpha_{0}^{(s)}}{\mathrm{d} z^{*}}$.

The solution satisfying the requirement that the radial component of the Lagrangian displacement remains finite at $r=R$, is

$$
\begin{aligned}
w_{0}^{(s)}\left(z^{*}\right)= & C_{0, s} z^{*}+D_{0, s} z^{*-1} \\
& -\frac{1}{2} z^{*} \alpha_{0}^{(s)}\left(z^{*}\right)+\frac{1}{2} \frac{1}{z^{*}} \int_{0}^{z^{*}} z^{\prime 2} \frac{\mathrm{d} \alpha_{0}^{(s)}}{\mathrm{d} z^{\prime}} \mathrm{d} z^{\prime},
\end{aligned}
$$

where $C_{0, s}$ en $D_{0, s}$ are general constants. The particular solution of the inhomogeneous equation can be transformed by partial integration and use of the first recurrence Relation (61), so that

$w_{0}^{(s)}\left(z^{*}\right)=A_{0, s} z^{*-\left(n_{\mathrm{e}}+1\right)} J_{n_{\mathrm{e}}}\left(z^{*}\right)+C_{0, s} z^{*}+D_{0, s} z^{*-1}$. 
By setting $\mu_{1}^{(s)}(\varepsilon)=\varepsilon \mu_{0}^{(s)}(\varepsilon)$ and $v_{1}^{(s)}(\varepsilon)=\varepsilon v_{0}^{(s)}(\varepsilon)$, one obtains the equations

$\frac{\mathrm{d}^{2} \alpha_{1}^{(s)}}{\mathrm{d} z^{* 2}}+\frac{2 n_{\mathrm{e}}+3}{z^{*}} \frac{\mathrm{d} \alpha_{1}^{(s)}}{\mathrm{d} z^{*}}+\alpha_{1}^{(s)}=0$,

$\frac{\mathrm{d}^{2} w_{1}^{(s)}}{\mathrm{d} z^{* 2}}+\frac{1}{z^{*}} \frac{\mathrm{d} w_{1}^{(s)}}{\mathrm{d} z^{*}}-\frac{1}{z^{* 2}} w_{1}^{(s)}=-\frac{\mathrm{d} \alpha_{1}^{(s)}}{\mathrm{d} z^{*}}$,

whose solutions can be written as

$$
\begin{aligned}
\alpha_{1}^{(s)}\left(z^{*}\right)= & A_{1, s} z^{*-\left(n_{\mathrm{e}}+1\right)} J_{n_{\mathrm{e}}+1}\left(z^{*}\right), \\
w_{1}^{(s)}\left(z^{*}\right)= & A_{1, s} z^{*-\left(n_{\mathrm{e}}+1\right)} J_{n_{\mathrm{e}}}\left(z^{*}\right) \\
& +C_{1, s} z^{*}+D_{1, s} z^{*-1}
\end{aligned}
$$

After renaming the sums of constants $A_{0, s}+\varepsilon A_{1, s}, C_{0, s}+$ ${ }_{\varepsilon} C_{1, s}, D_{0, s}+\varepsilon D_{1, s}$ respectively as $A_{0, s}, C_{0, s}, D_{0, s}$ and associating distinct functions $\mu_{0}^{(s)}(\varepsilon)$ with the three independent particular solutions, one obtains boundary-layer expansions valid from $r=R$ in the form

$$
\begin{gathered}
\alpha^{(s)}(r ; \varepsilon)=\mu_{0}^{(s, 1)}(\varepsilon) A_{0, s} z^{*-\left(n_{\mathrm{e}}+1\right)} J_{n_{\mathrm{e}}+1}\left(z^{*}\right), \\
w^{(s)}(r ; \varepsilon)=\varepsilon \mu_{0}^{(s, 1)}(\varepsilon) A_{0, s} z^{*-\left(n_{\mathrm{e}}+1\right)} J_{n_{\mathrm{e}}}\left(z^{*}\right) \\
\quad+\varepsilon \mu_{0}^{(s, 2)}(\varepsilon) C_{0, s} z^{*}+\varepsilon \mu_{0}^{(s, 3)}(\varepsilon) D_{0, s} z^{*-1}
\end{gathered}
$$

These boundary-layer expansions involve the two yet undetermined functions $\mu_{0}^{(s, 1)}(\varepsilon)$ and $\mu_{0}^{(s, 2)}(\varepsilon)$, and the three additional general constants $A_{0, s}, C_{0, s}$, and $D_{0, s}$. As for the boundary-layer expansions $\alpha^{(c)}(r ; \varepsilon)$ and $w^{(c)}(r ; \varepsilon)$, boundary-layer expansion $\alpha^{(s)}(r ; \varepsilon)$ is purely oscillatory, while boundary-layer expansion $w^{(s)}(r ; \varepsilon)$ contains a non-oscillatory part besides its oscillatory part.

\section{Matching of the boundary-layer expansions valid from $r=R$}

The boundary-layer expansions $\alpha^{(s)}(r ; \varepsilon)$ and $\xi^{(s)}(r ; \varepsilon)$ are also matched to the asymptotic expansions $\alpha^{(o)}(r ; \varepsilon)$ and $\xi^{(o)}(r ; \varepsilon)$ valid at larger distances from the singular boundary points.

The matching condition relative to the divergence of the Lagrangian displacement is similar to Condition (69). In this context, it is convenient to decompose the fast independent variable $\tau(r)$ as

$\tau(r)=\tau_{R}-\tau_{s}(r)$

where $\tau_{R}=\tau(R)$ and

$\tau_{s}(r)=\frac{1}{\varepsilon} \int_{0}^{z} \frac{\mathrm{d} z^{\prime}}{c\left(r^{\prime}\right)}$

For small values of $z$, the fast independent variable $\tau_{s}(r)$ tends to the boundary-layer coordinate $z^{*}(z)$.

The function $F(r)$ in asymptotic expansion $\alpha^{(o)}(r ; \varepsilon)$ is now decomposed as

$F(r)=F^{(s)}(z)-F^{(s)}\left(z_{0}\right)$ with

$$
\begin{aligned}
F^{(s)}(z)= & -c_{s} \frac{\left(2 n_{\mathrm{e}}+1\right)\left(2 n_{\mathrm{e}}+3\right)}{16 z^{1 / 2}}-\frac{1}{2} \int_{0}^{z}\left[c\left(r^{\prime}\right) W\left(r^{\prime}\right)\right. \\
& \left.+c_{s} \frac{\left(2 n_{\mathrm{e}}+1\right)\left(2 n_{\mathrm{e}}+3\right)}{16 z^{\prime 3 / 2}}\right] \mathrm{d} z^{\prime},
\end{aligned}
$$

so that, for small values of $z$,

$$
\begin{aligned}
F(r)=-c_{s} & \frac{\left(2 n_{\mathrm{e}}+1\right)\left(2 n_{\mathrm{e}}+3\right)}{16 z^{1 / 2}}-F^{(s)}\left(z_{0}\right) \\
+ & O\left(z^{1 / 2}\right) .
\end{aligned}
$$

Considering also that, for small values of $z$,

$h(r)=h_{s} z^{-\left(n_{\mathrm{e}}+3 / 2\right) / 2}$,

one has that

$$
\begin{aligned}
& \lim _{z \rightarrow 0} \alpha^{(o)}(r ; \varepsilon)=h_{s} z^{-\left(n_{\mathrm{e}}+3 / 2\right) / 2} \\
& \left\{A_{0}^{*} \cos \left(\tau_{R}-z^{*}\right)+B_{0}^{*} \sin \left(\tau_{R}-z^{*}\right)\right. \\
& -\varepsilon\left[c_{s} \frac{\left(2 n_{\mathrm{e}}+1\right)\left(2 n_{\mathrm{e}}+3\right)}{16 z^{1 / 2}}+F^{(s)}\left(z_{0}\right)\right] \\
& \left.\left[B_{0}^{*} \cos \left(\tau_{R}-z^{*}\right)-A_{0}^{*} \sin \left(\tau_{R}-z^{*}\right)\right]\right\} .
\end{aligned}
$$

On the other hand, one has that

$\lim _{z \rightarrow \infty} \alpha^{(s)}(r ; \varepsilon)=\mu_{0}^{(s, 1)}(\varepsilon) \varepsilon^{n_{\mathrm{e}}+3 / 2} A_{0, s} E_{s} z^{-\left(n_{\mathrm{e}}+3 / 2\right) / 2}$

$$
\begin{aligned}
& {\left[\sin \left(z^{*}-\chi\right)\right.} \\
& \left.\quad+\varepsilon c_{s} \frac{\left(2 n_{\mathrm{e}}+1\right)\left(2 n_{\mathrm{e}}+3\right)}{16 z^{1 / 2}} \cos \left(z^{*}-\chi\right)\right]
\end{aligned}
$$

with $\chi=\left(2 n_{\mathrm{e}}+1\right) \pi / 4, E_{s}=(2 / \pi)^{1 / 2}\left(2 / c_{s}\right)^{-\left(n_{\mathrm{e}}+3 / 2\right)}$.

A matching of the boundary-layer expansion $\alpha^{(s)}(r ; \varepsilon)$ to the asymptotic expansion $\alpha^{(o)}(r ; \varepsilon)$ is possible when

$\mu_{0}^{(s, 1)}(\varepsilon)=\varepsilon^{-\left(n_{\mathrm{e}}+3 / 2\right)}$,

and the coefficients of $\cos z^{*}$ and $\sin z^{*}$ are identically zero, so that

$$
\left.\begin{array}{l}
A_{0}^{*} \cos \tau_{R}+B_{0}^{*} \sin \tau_{R} \\
-\varepsilon\left[c_{s} \frac{\left(2 n_{\mathrm{e}}+1\right)\left(2 n_{\mathrm{e}}+3\right)}{16 z^{1 / 2}}+F^{(s)}\left(z_{0}\right)\right] \\
\left(B_{0}^{*} \cos \tau_{R}-A_{0}^{*} \sin \tau_{R}\right)=-A_{0, s} \frac{E_{s}}{h_{s}} \\
{\left[\sin \chi-\varepsilon c_{s} \frac{\left(2 n_{\mathrm{e}}+1\right)\left(2 n_{\mathrm{e}}+3\right)}{16 z^{1 / 2}} \cos \chi\right],} \\
A_{0}^{*} \sin \tau_{R}-B_{0}^{*} \cos \tau_{R} \\
-\varepsilon\left[c_{s} \frac{\left(2 n_{\mathrm{e}}+1\right)\left(2 n_{\mathrm{e}}+3\right)}{16 z^{1 / 2}+F^{(s)}}\left(z_{0}\right)\right] \\
\left(B_{0}^{*} \sin \tau_{R}+A_{0}^{*} \cos \tau_{R}\right)=A_{0, s} \frac{E_{s}}{h_{s}} \\
{\left[\cos \chi+\varepsilon c_{s} \frac{\left(2 n_{\mathrm{e}}+1\right)\left(2 n_{\mathrm{e}}+3\right)}{16 z^{1 / 2}} \sin \chi\right] .}
\end{array}\right\}
$$


As, at order $\varepsilon^{0}$,

$$
\left.\begin{array}{c}
A_{0}^{*}=A_{0, s} \frac{E_{s}}{h_{s}} \sin \left(\tau_{R}-\chi\right), \\
B_{0}^{*}=-A_{0, s} \frac{E_{s}}{h_{s}} \cos \left(\tau_{R}-\chi\right),
\end{array}\right\},
$$

Eqs. (107) reduce to

$$
\left.\begin{array}{c}
A_{0}^{*}=A_{0, s} \frac{E_{s}}{h_{s}} \\
{\left[\sin \left(\tau_{R}-\chi\right)-\varepsilon F^{(s)}\left(z_{0}\right) \cos \left(\tau_{R}-\chi\right)\right],} \\
B_{0}^{*}=-A_{0, s} \frac{E_{s}}{h_{s}} \\
\quad\left[\cos \left(\tau_{R}-\chi\right)+\varepsilon F^{(s)}\left(z_{0}\right) \sin \left(\tau_{R}-\chi\right)\right] .
\end{array}\right\}
$$

The non-oscillatory part of the asymptotic expansion $\xi^{(o)}(r ; \varepsilon)$ tends to that of the boundary-layer expansion $\xi^{(s)}(r ; \varepsilon)$ as $z \rightarrow 0$, when

$C_{0, s}=0$

and

$\mu_{0}^{(s, 3)}(\varepsilon)=\varepsilon^{-1}, \quad C_{0}^{*}=D_{0, s} R^{-(\ell-1)} \frac{c_{s}^{2}}{2}$.

Because of the second Eq. (82), it follows that

$C_{0, c}=\frac{1}{2} D_{0, s} R^{-(\ell-1)} c_{c}^{\ell-2} c_{s}^{2}$

By the matching, the functions $\mu_{0}^{(s, 1)}(\varepsilon)$ and $\mu_{0}^{(s, 3)}(\varepsilon)$ are determined, the constants $A_{0}^{*}$ and $B_{0}^{*}$ are related to the constant $A_{0, s}$, and the constant $C_{0, c}$ is related to the constant $D_{0, s}$.

At this point, three constants remain undetermined: $A_{0, c}$, $A_{0, s}$, and $D_{0, s}$. They are determined in the next two sections.

\section{The eigenfrequency equation}

Elimination of the constants $A_{0}^{*}$ and $B_{0}^{*}$ from Eqs. (80) and (109) leads to a homogeneous system of two equations for the constants $A_{0, c}$ and $A_{0, s}$. A necessary and sufficient condition for the system to admit of a non-trivial solution is

$\tan \left(\frac{\ell \pi}{2}+\chi-\tau_{R}\right)=-\varepsilon T_{\ell}$

where $T_{\ell}$ is a constant defined as

$T_{\ell}=F^{(s)}\left(z_{0}\right)-F^{(c)}\left(r_{0}\right)$.

It follows that

$\tau_{R} \equiv|\sigma| \int_{0}^{R} \frac{\mathrm{d} r}{c(r)}=\left(2 n+\ell+n_{\mathrm{e}}+\frac{1}{2}\right) \frac{\pi}{2}+\frac{T_{\ell}}{|\sigma|}$

with $n=1,2,3, \ldots$ This equation corresponds to the eigenfrequency equation derived by Tassoul (1990). The relation between $n$ and the radial order of the mode considered is determined in Sect. 11.
The value of the constant $T_{\ell}$ is independent of the choice of the point with coordinates $r_{0}$ and $z_{0}$, since, on the grounds of Definitions (73) and (101), the constant can be expressed as

$$
\begin{aligned}
T_{\ell}=- & \frac{1}{2}\left\{\int_{0}^{R} I(r) \mathrm{d} r\right. \\
& \left.+c_{c} \frac{\ell(\ell+1)}{R}+c_{s} \frac{\left(2 n_{\mathrm{e}}+1\right)\left(2 n_{\mathrm{e}}+3\right)}{8 R^{1 / 2}}\right\}
\end{aligned}
$$

with

$$
\begin{aligned}
I(r)= & c(r) W(r) \\
& +c_{c} \frac{\ell(\ell+1)}{r^{2}}+c_{s} \frac{\left(2 n_{\mathrm{e}}+1\right)\left(2 n_{\mathrm{e}}+3\right)}{16(R-r)^{3 / 2}} .
\end{aligned}
$$

\section{The condition on the Eulerian perturbation of the gravitational potential at $r=R$}

The last undetermined constant, $D_{0, s}$, is fixed by boundary Condition (9) on the Eulerian perturbation of the gravitational potential at $r=R$. In order to impose the condition, we use the following two equations, which express the Eulerian perturbation of the gravitational perturbation and its first derivative in terms of the divergence $\alpha(r)$ and the radial component $\xi(r)$ of the Lagrangian displacement:

$$
\begin{aligned}
\Phi^{\prime}= & \left(c^{2} \alpha-g \xi\right) \\
& +\varepsilon^{2} \frac{r^{2}}{\ell(\ell+1)}\left[\frac{1}{r^{2}} \frac{\mathrm{d}}{\mathrm{d} r}\left(r^{2} \xi\right)-\alpha\right], \\
\frac{\mathrm{d} \Phi^{\prime}}{\mathrm{d} r}= & \frac{\mathrm{d}\left(c^{2} \alpha\right)}{\mathrm{d} r}-\frac{N^{2}}{g} c^{2} \alpha-g \frac{\mathrm{d} \xi}{\mathrm{d} r} \\
& +\left(2 \frac{g}{r}-4 \pi G \rho\right) \xi+\varepsilon^{2} \xi
\end{aligned}
$$

(Smeyers et al. 1995). The boundary condition then takes the form

$\frac{1}{\varepsilon^{2}} \frac{R}{\ell}\left[\left(\frac{\mathrm{d} \xi}{\mathrm{d} r}\right)_{R}+\frac{\ell+2}{R} \xi_{R}-\alpha_{R}\right]+O\left(\varepsilon^{0}\right)=0$.

The coefficient of the term of the order $\varepsilon^{-2}$ is identically zero for the dominant oscillatory parts in the boundary-layer expansions $\alpha^{(s)}(r ; \varepsilon)$ and $\xi^{(s)}(r ; \varepsilon)$. It also becomes equal to zero for the non-oscillatory part in the boundary-layer expansion $\xi^{(s)}(r ; \varepsilon)$, when one sets

$D_{0, s}=0$.

Because of the second Eq. (111), it follows that

$C_{0}^{*}=0, \quad C_{0, c}=0$.

Consequently, the asymptotic representation of the radial component of the Lagrangian displacement is purely oscillatory in the interval $[0,1]$ as well as that of the divergence of the Lagrangian displacement. 


\section{Uniformly valid asymptotic expansions}

Since all constants are now fixed, it is possible to construct the uniformly valid asymptotic expansions for the divergence and the radial component of the Lagrangian displacement in a final form. One obtains them by adding the boundary-layer expansion and the expansion valid at larger distances from the boundary point considered, and subtracting the part common to both expansions. In the uniformly valid asymptotic expansions presented here, we have expressed the constants $A_{0}^{*}$ and $B_{0}^{*}$ respectively in terms of the constants $A_{0, c}$ and $A_{0, s}$ by means of Eqs. (80) and (109).

The asymptotic expansions that are uniformly valid from $r=0$ to a sufficiently large distance from $r=R$ can be expressed as

$$
\begin{aligned}
& \alpha^{(c, u)}(r ; \varepsilon)=A_{0, c}\left\{\varepsilon^{-1} r^{*-1 / 2} J_{\ell+1 / 2}\left(r^{*}\right)\right. \\
& +\left(\frac{2}{\pi}\right)^{1 / 2} \frac{c_{c}}{h_{c}} h(r) \\
& {\left[\sin \left(\tau-\frac{\ell \pi}{2}\right)+\varepsilon\left(F(r)+F^{(c)}\left(r_{0}\right)\right) \cos \left(\tau-\frac{\ell \pi}{2}\right)\right]} \\
& -\left(\frac{2}{\pi}\right)^{1 / 2} \frac{c_{c}}{r}\left[\sin \left(r^{*}-\frac{\ell \pi}{2}\right)\right. \\
& \left.\left.+\varepsilon c_{c} \frac{\ell(\ell+1)}{2 r} \cos \left(r^{*}-\frac{\ell \pi}{2}\right)\right]\right\}, \\
& \xi^{(c, u)}(r ; \varepsilon)=A_{0, c} \\
& \left\{-\frac{c_{c}}{2 \ell+1} r^{*-1 / 2}\left[\ell J_{\ell-1 / 2}\left(r^{*}\right)-(\ell+1) J_{\ell+3 / 2}\left(r^{*}\right)\right]\right. \\
& -\varepsilon\left(\frac{2}{\pi}\right)^{1 / 2} \frac{c_{c}}{h_{c}} c(r) h(r) \\
& +\varepsilon\left(\frac{2}{\pi}\right)^{1 / 2} \frac{c_{c}^{2}}{r}\left\{\cos \left(r^{*}-\frac{\ell \pi}{2}\right)\right. \\
& \left.\cos \left(\tau-\frac{\ell \pi}{2}\right)-\varepsilon\left(G(r)+F^{(c)}\left(r_{0}\right)\right) \sin \left(\tau-\frac{\ell \pi}{2}\right)\right]
\end{aligned}
$$

Furthermore, the asymptotic expansions that are uniformly valid from $r=R$ to a sufficiently large distance from $r=0$, can be expressed as

$$
\begin{aligned}
& \alpha^{(s, u)}(r ; \varepsilon)=A_{0, s}\left\{\varepsilon^{-\left(n_{\mathrm{e}}+3 / 2\right)} z^{*-\left(n_{\mathrm{e}}+1\right)} J_{n_{\mathrm{e}}+1}\left(z^{*}\right)\right. \\
& +\frac{E_{s}}{h_{s}} h(r)\left[\sin \left(\tau_{s}-\chi\right)\right. \\
& \left.-\varepsilon\left(F(r)+F^{(s)}\left(z_{0}\right)\right) \cos \left(\tau_{s}-\chi\right)\right] \\
& -E_{s} z^{-\left(n_{\mathrm{e}}+3 / 2\right) / 2}\left[\sin \left(z^{*}-\chi\right)\right. \\
& \left.\left.+\varepsilon c_{s} \frac{\left(2 n_{\mathrm{e}}+1\right)\left(2 n_{\mathrm{e}}+3\right)}{16 z^{1 / 2}} \cos \left(z^{*}-\chi\right)\right]\right\}
\end{aligned}
$$

$$
\begin{aligned}
& \xi^{(s, u)}(r ; \varepsilon)=A_{0, s}\left\{\varepsilon^{-\left(n_{\mathrm{e}}-1 / 2\right)} \frac{c_{s}^{2}}{2} z^{*-n_{\mathrm{e}}} J_{n_{\mathrm{e}}}\left(z^{*}\right)\right. \\
& +\varepsilon \frac{E_{s}}{h_{s}} c(r) h(r) \\
& {\left[\cos \left(\tau_{s}-\chi\right)+\varepsilon\left(G(r)+F^{(s)}\left(z_{0}\right)\right) \sin \left(\tau_{s}-\chi\right)\right]} \\
& -\varepsilon E_{s} c_{s} z^{-\left(n_{\mathrm{e}}+1 / 2\right) / 2}\left[\cos \left(z^{*}-\chi\right)\right. \\
& \left.\left.-\varepsilon c_{s} \frac{\left(2 n_{\mathrm{e}}-1\right)\left(2 n_{\mathrm{e}}+1\right)}{16 z^{1 / 2}} \sin \left(z^{*}-\chi\right)\right]\right\}
\end{aligned}
$$

\section{Identification of the radial order of a mode associated with a given eigenfrequency}

The radial order of a mode, radial or non-radial, associated with a given eigenfrequency is determined by the number of nodes displayed by the eigenfunction $\xi(r)$. For the determination of this number, it suffices to consider the lowest-order uniformly valid solutions for $\xi(r)$. For convenience, we express them in the compact forms

$$
\begin{aligned}
& \xi^{(c, u)}(r ; \varepsilon)=-\varepsilon \frac{A_{0, c}}{2 \ell+1} \frac{c_{c}}{h_{c}} c(r) h(r) \\
& \tau^{1 / 2}\left[\ell J_{\ell-1 / 2}(\tau)-(\ell+1) J_{\ell+3 / 2}(\tau)\right] \\
& \xi^{(s, u)}(r ; \varepsilon)=\varepsilon A_{0, s}\left(\frac{2}{c_{s}}\right)^{-\left(n_{\mathrm{e}}+3 / 2\right)} c(r) h(r) \\
& \tau_{s}^{1 / 2} J_{n_{\mathrm{e}}}\left(\tau_{s}\right) .
\end{aligned}
$$

For a radial mode, the number of nodes in the eigenfunction $\xi(r)$ can be determined with the procedure of Ledoux (1962). In the case of radial modes, only the Bessel function $J_{3 / 2}(\tau)$ appears in the asymptotic solution $\xi^{(c, u)}(r ; \varepsilon)$. According to McMahon's expansions for the zeros of Bessel functions, it has zeros approximatively at

$$
\tau^{0}=(2 j+1) \frac{\pi}{2}, \quad j=1,2,3, \ldots
$$

The largest admissible value of $j$ is fixed by the condition that $\tau^{0} \leq \tau_{R}$, so that

$2 j+1 \leq 2 n+n_{\mathrm{e}}+\frac{1}{2}$.

When $n_{\mathrm{e}}<1 / 2$, it follows that $j<n$, so that $n-1$ is the largest admissible value of $j$. When $n_{\mathrm{e}} \geq 1 / 2$, one must consider the position of the first zero of the Bessel function $J_{n_{\mathrm{e}}}\left(\tau_{s}\right)$, which appears in the asymptotic solution $\xi^{(s, u)}(r ; \varepsilon)$ and has zeros approximatively at

$$
\tau_{s}^{0}=\left(2 r+n_{\mathrm{e}}-\frac{1}{2}\right) \frac{\pi}{2}, \quad r=1,2,3, \ldots
$$

With the first node counted from $\tau_{s}=0$ coincides the last node counted from $\tau=0$. Therefore, the position of this last node is given by

$\tau^{0}=\tau_{R}-\tau_{s}^{0}=(2 n-1) \frac{\pi}{2}$ 


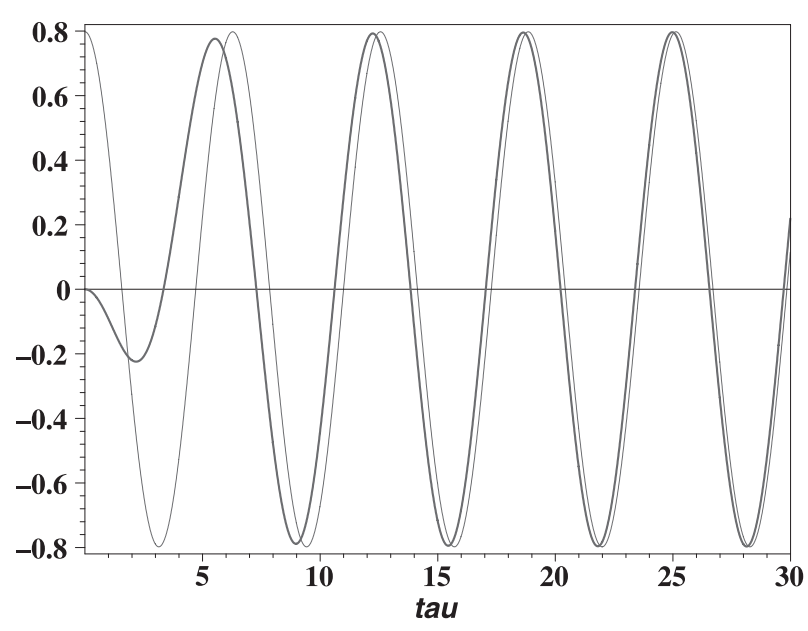

Fig. 1. The function $H(\tau)$ (thick line) and its asymptotic form $H_{\text {asymp }}(\tau)$ for large values of $\tau$ (thin line).

Again, $n-1$ is the largest admissible value of $j$. Hence, in both cases, the asymptotic eigenfunction $\xi(r)$ displays $n-1$ nodes. For conclusion, the radial mode associated with a number $n$ in eigenfrequency Eq. (115) is the $(n-1)$ th overtone.

For the determination of the number of nodes in the asymptotic eigenfunction $\xi(r)$ of a non-radial mode $p$, we consider the case of $\ell=2$ as an example.

With regard to the asymptotic solution $\xi^{(c, u)}(r ; \varepsilon)$, it is appropriate to consider the function

$H(\tau) \equiv \frac{\tau^{1 / 2}}{2 \ell+1}\left[2 J_{3 / 2}(\tau)-3 J_{7 / 2}(\tau)\right]$

and to compare the positions of its zeros with those of its asymptotic form that is valid for large values of $\tau$

$H_{\text {asymp }}(\tau) \equiv-\left(\frac{2}{\pi}\right)^{1 / 2} \cos \tau$.

The latter function has zeros at

$\tau^{0}=(2 j+1) \frac{\pi}{2}, \quad j=0,1,2, \ldots$

The variations of the functions $H(\tau)$ and $H_{\text {asymp }}(\tau)$ are represented in Fig. 1. It appears that the zero of the asymptotic function $H_{\text {asymp }}(\tau)$ associated with $j=0$ does not correspond to a zero of the function $H(\tau)$, so that the zeros of the function $H(\tau)$ must be counted from $j=1$.

In the asymptotic solution $\xi^{(s, u)}(r ; \varepsilon)$, the Bessel function $J_{n_{\mathrm{e}}}\left(\tau_{s}\right)$ has zeros approximatively at

$\tau_{s}^{0}=\left(2 k+n_{\mathrm{e}}-\frac{1}{2}\right) \frac{\pi}{2}, \quad k=1,2,3, \ldots$

The position $\tau_{s}^{0}$ of the first zero yields for the position of the last zero counted from $\tau=0$

$\tau^{0}=\tau_{R}-\tau_{s}^{0}=(2 n+1) \frac{\pi}{2}$.

Hence, for a non-radial mode $p$ associated with a number $n$ in eigenfrequency Eq. (115), the eigenfunction $\xi(r)$ displays $n$ nodes, and the mode is of radial order $n$ according to Cowling's classification.
The number of nodes of the asymptotic eigenfunction $\alpha(r)$ can be determined in a similar way. The compact forms of the uniformly valid asymptotic solutions are

$$
\begin{aligned}
\alpha^{(c, u)}(r ; \varepsilon)= & A_{0, c} c_{c} \frac{h(r)}{h_{c}} \tau^{1 / 2} J_{\ell+1 / 2}(\tau), \\
\alpha^{(s, u)}(r ; \varepsilon)= & A_{0, s}\left(\frac{2}{c_{s}}\right)^{-\left(n_{\mathrm{e}}+3 / 2\right)} \frac{h(r)}{h_{s}} \\
& \times \tau_{s}^{1 / 2} J_{n_{\mathrm{e}}+1}\left(\tau_{s}\right) .
\end{aligned}
$$

One verifies that, for any mode, radial or non-radial, the asymptotic eigenfunction $\alpha(r)$ displays $n-1$ nodes, i.e. the same number of nodes as the eigenfunction $\xi(r)$ in case of a radial mode, and one node less than the eigenfunction $\xi(r)$ in case of a nonradial $p$-mode.

\section{Concluding remarks}

The present asymptotic analysis confirms that the application of a two-variable expansion procedure and boundary-layer theory to the system of Eqs. (3) and (4) is particularly appropriate for the construction of the asymptotic representation of higherorder $p$-modes in a spherically symmetric star.

The dependent variable $\alpha(r)$ appears to be the basic function. In each region, the lowest-order asymptotic approximation of $\alpha(r)$ is determined by a homogeneous second-order differential equation.

We have improved the asymptotic theory of Smeyers et al. (1996) in two ways.

First, we have incorporated the function $v(\varepsilon)$ in the asymptotic expansion $\xi^{(o)}(r ; \varepsilon)$ for the radial component of the Lagrangian displacement valid at larger distances from the singular boundary points. This function is determined in such a way that already the lowest-order approximation in expansion $\xi^{(o)}(r ; \varepsilon), \xi^{(o)}(\tau, r)$, is solution of an inhomogeneous differential equation, so that it contains an oscillatory part besides the nonoscillatory part. From the determination of the function $v(\varepsilon)$, it is manifest that the expansion $\xi^{(o)}(r ; \varepsilon)$ for the radial component of the Lagrangian displacement is of one order higher in the small expansion parameter $\varepsilon$ than expansion $\alpha^{(o)}(r ; \varepsilon)$ for the divergence of the Lagrangian displacement. The further analysis shows that $\xi(r)$ is even everywhere of one order higher than $\alpha(r)$.

Secondly, in the boundary layers, we have no longer adopted a coordinate that is identical to the fast variable used in the two-variable expansion procedure at larger distances, but we have followed the usual prescriptions. The boundary-layer coordinate then corresponds to the first term in the Taylor expansion of the fast variable from the singular boundary point considered. With this procedure, the matching conditions for the boundary-layer expansions and the asymptotic expansions valid at larger distances have the simple form of Eq. 69), and the constructions of the uniformly valid asymptotic expansions are more transparent.

The validity of the present asymptotic theory, as well as that of Tassoul's asymptotic theory, is restricted by the condition that $\varepsilon \ll 1$, i.e. that the oscillation period is much smaller than $2 \pi$ times the star's dynamic time scale $\left[R^{3} /(G M)\right]^{1 / 2}$. For 
example, in the case of the Sun, the oscillation period must be much shorter than nearly $10^{4} \mathrm{~s}$ or $2 \mathrm{~h} 47 \mathrm{~min}$.

A second restriction on the validity of the asymptotic theory is related to the dominant boundary-layer Eq. (56). This equation is derived from Eq. (3) in the supposition that the term $\sigma^{2} / c^{2}(r)$ becomes of the same order of magnitude as the term $-\ell(\ell+1) / r^{2}$ somewhere in the boundary layer near $r=0$. Physically, the supposition implies that, for the angular frequency considered, the inner boundary of the acoustic resonant cavity is sufficiently close to the star's centre. This condition is violated for smaller values of $\sigma$ and for larger values of the degree $\ell$ of the spherical harmonic. In these cases, the term $\sigma^{2} / c^{2}(r)$ must no longer be incorporated in the dominant boundary-layer equation. An asymptotic theory applying to them was developed by Smeyers (2003).

In the lowest-order asymptotic approximation, it follows from the uniformly valid asymptotic Solutions (127) and (128) that

$\frac{\mathrm{d} \xi(r)}{\mathrm{d} r}=\alpha(r)$

everywhere in a star. Equation (3) can then be written as

$$
\begin{aligned}
& \frac{\mathrm{d}}{\mathrm{d} r}\left(r^{2} \rho c^{4} \frac{\mathrm{d} \alpha}{\mathrm{d} r}\right) \\
&+\left[\frac{1}{\varepsilon^{2}} \frac{1}{c^{2}}+K_{3}(r)+K_{4}(r)\right] r^{2} \rho c^{4} \alpha=0,
\end{aligned}
$$

so that it strictly reduces to a homogeneous second-order differential equation of the Sturm-Liouville type. This property explains that the eigenfunction for $\alpha(r)$ that is associated with the $n$th eigenfrequency displays $n-1$ nodes, with $n=1,2,3, \ldots$

Acknowledgements. The author acknowledges helpful remarks from an unknown referee and from Dr. M. Tassoul.

\section{References}

Abramowitz, M., \& Stegun, I. A. 1965, Handbook of Mathematical Functions (New York: Dover)

Kevorkian, J., \& Cole, J. D. 1981, Perturbation Methods in Applied Mathematics (New York: Springer)

Kevorkian, J., \& Cole, J. D. 1996, Multiple Scale and Singular Perturbation Methods (New York: Springer)

Ledoux, P. 1962, Bull. Acad. Roy. Belg. Cl. Sc. 5e Série, 48, 240

Olver, F. W. J. 1974, Asymptotics and Special Functions (New York: Academic Press)

Pekeris, C. L. 1938, ApJ, 88, 189

Smeyers, P. 2003, A\&A, 407, 643

Smeyers, P., De Boeck, I., Van Hoolst, T., \& Decock, L. 1995, A\&A, 301, 105

Smeyers, P., Vansimpsen, T., De Boeck, I., \& Van Hoolst, T. 1996, A\&A, 307, 105

Tassoul, M. 1990, ApJ, 358, 313 